\title{
Determinantes da evolução do peso e altura em crianças de 3 meses a 6 anos assistidas em creche: análise por modelo linear não hierarquizado em ensaio quase-experimental
}

\author{
Ana Maria Segall-Corrêa, ${ }^{1}$ Neusa Nunes da Silva e Gonçalves, ${ }^{1}$ \\ Liciana Vaz Arruda Silveira Chalita, ${ }^{2}$ Gabriela Picarelli Russo-Leite, ${ }^{1}$ \\ Carlos Roberto Padovani ${ }^{2}$ e Aguinaldo Gonçalves ${ }^{3}$
}

RESUMO Objetivo. Avaliar os determinantes do crescimento em crianças de 3 meses a 6 anos assistidas em creche usando como grupo de comparação crianças de características semelhantes mas que não freqüentavam creche.

Métodos. Foi planejado um estudo quase experimental com observação de 444 crianças entre 3 e 72 meses de idade, residentes em área periférica de Sorocaba, Estado de São Paulo, Brasil, constituindo dois grupos: o grupo de intervenção (164 crianças matriculadas em creche) e o grupo de não intervenção (280 que não freqüentavam creche). Ambos os grupos foram acompanhados por 16 meses, mediante quatro observações. Foram medidos o peso e a altura e coletadas informações sobre antecedentes sócio-demográficos e de morbidade da mãe, características das crianças ao nascimento, morbidade nos últimos 15 dias antes de cada observação e hospitalizações.

Resultados. Os lactentes e pré-escolares dos dois grupos eram de famílias de baixo poder aquisitivo, 65\% com renda média mensal inferior a 100 dólares. Oitenta por cento das mães tinham ensino fundamental (8 anos) ou menor. A regressão linear multivariada mostrou que na primeira observação - portanto, antes da entrada na creche - o peso ao nascer foi a única condição a explicar as variações nutricionais dos grupos. Modelagens subseqüentes revelaram que a freqüência ou não à creche é condição determinante do crescimento, especialmente quando se analisa a adequação do peso para idade, tendo sido controlados os efeitos do peso ao nascer, sexo, idade ao início do estudo e doenças nos 15 dias anteriores às entrevistas. O impacto nutricional da freqüência à creche já era significativo nos primeiros 3 meses de observação.

Conclusões. O benefício nutricional da assistência prestada nas creches supera os efeitos negativos da maior morbidade observada entre crianças que freqüentam creche.

Palavras chave Nutrição infantil, crescimento infantil, peso, altura, Brasil.

1 Universidade Estadual de Campinas (UNICAMP), Departamento de Medicina Preventiva e Social, Área de Epidemiologia. Correspondência e pedidos de separatas devem ser enviados a Ana Maria Segall-Corrêa no seguinte endereço: Rua Almirante Noronha 157, Cidade Universitária II, CEP 13083-030, Campinas, SP, Brasil. Fone: +55-193287-4107; e-mail: segall@lexxa.com.br

2 Universidade Estadual de São Paulo em Botucatu (UNESP), Instituto de Biociências, Departamento de Bioestatística, Botucatu, São Paulo, Brasil.
A falta de nutrição adequada ainda está relacionada diretamente a 55\% das mortes infantis em todo o mundo (1). No Brasil, a redução da desnu-

3 UNICAMP, Faculdade de Educação Física, Grupo de Saúde Coletiva e Atividade Física. trição infantil verificada nas últimas décadas tem sido documentada em análise de tendência histórica, especialmente a partir de dois inquéritos nacionais sobre saúde e nutrição realizados em 1975 e 1989 (2). Esses inquéritos mostraram que, considerando o indicador peso para idade, a desnu- 
trição caiu 61\%, de 18\% em 1975 para $7 \%$ em 1989. Entretanto, essa melhora específica tem a mesma característica de iniqüidade da distribuição de todos os benefícios sociais no Brasil, ou seja, persistem diferenças entre as regiões Norte e Nordeste em relação ao Sul e Sudeste, da mesma forma que as populações infantis mais pobres, residentes nas áreas rurais ou na periferia das cidades mais ricas, seguem mais sujeitas às carências e suas conseqüências.

A nutrição adequada de tais segmentos continua sendo um dos mais amplos desafios para as políticas públicas no Brasil. As iniciativas de assistência alimentar, tais como subsídios, distribuição de cestas básicas a famílias carentes ou suplemento alimentar a grupos de maior risco biológico, apesar de significativa expansão a partir da década de 1970 (3), não foram suficientes para fazer frente aos efeitos negativos das políticas de ajuste econômico desde então implementadas (4). O baixo impacto desses programas setoriais pode ser atribuído tanto à natureza excludente das diretrizes econômicas que penalizam os mais vulneráveis, quanto à sua abrangência, considerada insuficiente para atingir esse segmento (5). Apesar de representarem dispêndio monetário muito expressivo, os programas de assistência alimentar no Brasil, tais como os programas de suplementação alimentar, do Ministério da Saúde, o programa de alimentação do trabalhador, do Ministério do Trabalho, o de merenda escolar, do Ministério da Educação, e, mais recentemente, os programas de atendimento às emergências (distribuição de cestas básicas), não têm sido suficientemente avaliados (6).

Para muitos pré-escolares, a assistência alimentar em creches é um fator de segurança alimentar e, conseqüentemente, de bem estar nutricional. Dados oficiais recentes mostram que a merenda escolar (7) é responsável por 36 milhões de refeições diárias, oferecidas tanto a crianças do ensino fundamental quanto àquelas assistidas em creches públicas. Esses programas, portanto, podem constituir-se em instrumento de proteção quando se consideram os grupos mais pobres da popu- lação brasileira (8). Entretanto, deve-se ainda acrescentar que lactentes e préescolares que freqüentam instituições, especialmente as creches, estão, também, sujeitas a maior risco de contraírem doenças infecciosas, condições reconhecidas como associadas ao pior desempenho no crescimento. De fato, observações confirmam (9) que crianças mantidas em creches adoecem mais do que aquelas mantidas em seus domicílios. A prevalência de pneumonia pode ser de duas a 12 vezes maior, enquanto a da diarréia é 60 a $250 \%$ superior. O risco de adoecer mais por infecção respiratória aguda pode, ainda, passar de 3 para 5 quando a permanência em instituições eleva-se de 15 para 50 horas semanais (10).

Metodologicamente, essas duas tendências - a tendência a melhorar o estado nutricional e a tendência a contrair mais doenças infecciosas podem ser apuradas mediante monitoramento do crescimento, utilizando procedimentos antropométricos, preditores viáveis e seguros de estado de saúde, comprometimento funcional e mortalidade (11).

Por demanda de gestores municipais da Secretaria de Educação de Sorocaba, cidade de aproximadamente meio milhão de habitantes e distante 100 quilômetros da capital do Estado de São Paulo, cujo dispêndio com alimentação de pré-escolares chegava, em 1994, a 9 milhões de dólares, realizou-se, em 1995, investigação com o objetivo de avaliar o impacto da assistência alimentar em creche sobre o perfil de crescimento de crianças menores de 6 anos, descrita em estudo publicado em 1999 (12). A partir dos dados obtidos naquele estudo inicial, o objetivo do presente estudo foi avaliar os determinantes do impacto da assistência nutricional sobre o perfil de crescimento na mesma população.

\section{MATERIAIS E MÉTODOS}

Na fase de planejamento do estudo, identificaram-se, com o auxílio dos técnicos da Secretaria de Educação e Cultura de Sorocaba, duas creches inauguradas no ano letivo de 1995 e cujas populações das respectivas áreas de cobertura não haviam sido ainda beneficiárias de programas oficiais de suplementação alimentar. A presente comunicação refere-se às crianças de uma dessas creches, da Vila Sabiá e setores adjacentes (Vilas Zacarias e João Romão), por terem sido, apenas para essa creche, obtidos grupos de intervenção (GI) e não intervenção (GN) residentes na mesma área geográfica. A impossibilidade, por razões éticas, de garantir aleatoriedade no processo de alocação no GI ou GN, aliada à autoseleção para inscrição em lista de vagas e, também, a prioridade dada aos filhos de mães trabalhadoras, definiram este estudo como do tipo quaseexperimental (13).

A alocação das crianças no GI se deu mediante sorteio institucional para ingresso na creche a partir de lista de candidatos. Como os excedentes não eram em número suficiente para constituir o GN, optou-se por buscar nos domicílios as crianças da Vila Sabiá que, por qualquer motivo, não haviam se candidatado às vagas. Assim, foi realizado um censo, a fim de identificar aquelas elegíveis para inclusão no GN. Todas as crianças residentes na área, matriculadas ou não, com idade entre 3 meses e 7 anos incompletos eram elegíveis para o estudo.

Após esses procedimentos, foram alocadas para o estudo 532 crianças com idade entre 3 e 72 meses: 196 matriculadas (GI) e 336 não matriculadas (GN). Dessas, 444 foram acompanhadas: 164 no GI e 280 no GN. No GI, 29 foram excluídas da análise por terem sido observadas apenas uma vez, e outras três, por terem abandonado a creche ao longo do seguimento (total de 32 perdas). No GN, excluíram-se 54 por terem apenas uma observação, oito por mudança de condição de não exposição, já que foram matriculadas na creche, e duas por apresentarem problemas de consistência no registro de seus dados, com valores de escore $\mathrm{z}$ extremamente discrepantes (total de 64 perdas).

O acompanhamento dos dois grupos foi realizado entre 1995 e 1996, em quatro observações sucessivas, com intervalo de 3 meses entre si. Nessas 
ocasiões procedia-se à avaliação antropométrica (peso e estatura ou comprimento) e de antecedentes mórbidos, além de coleta, na primeira entrevista, de informações sobre antecedentes sociais, gestacionais e condições de nascimento dos participantes.

As entrevistadoras receberam treinamento prévio e a realização de medidas antropométricas foi padronizada (14). Usaram-se balanças eletrônicas portáteis do tipo plataforma, marca Filizola, com escala de $100 \mathrm{~g}$ para peso máximo de $150 \mathrm{~kg}$ e antropômetros horizontais (para crianças de até 2 anos) e verticais (para aquelas com idade acima de 2 anos), ambos com intervalo escalar de $10 \mathrm{~mm}$. Todos os equipamentos passaram por aferição no Instituto de Pesos e Medidas/Instituto Nacional de Metrologia (IPEM/ INMETRO), agência de Campinas.

No GI, as entrevistas referentes à primeira observação ocorreram logo após a matrícula. As entrevistas relativas à primeira observação no GN ocorreram 3 meses mais tarde, tendo em vista a necessidade de realizar um censo para selecionar crianças comparáveis às do GI. Os exames antropométricos da primeira observação foram realizados na creche para o GI e nos domicílios para o GN, passando ambos, nas observações seguintes, a serem realizados na creche, dada a precariedade das condições domiciliares, especialmente para verificação de altura com o aparelho vertical.

Menores de 2 anos eram pesados sem roupa, e os demais, vestindo uma peça de roupa íntima. Os antropômetros horizontais eram operados sobre a superfície plana de uma mesa. Fixavam-se os verticais (fitas antropométricas) em parede lisa, sem rodapé, seguindo as instruções dos fabricantes. Os equipamentos foram instalados em salas da creche, de acordo com o número de equipes.

Nos procedimentos de análise, utilizaram-se como indicadores do estado nutricional escores das relações peso para idade e peso para altura padronizados em unidades de desvios-padrão (DP) de afastamento da mediana da população de referência (11). Outros detalhamentos dos métodos e técnicas utilizadas estão disponíveis em publicações prévias $(12,15)$.

Para o estudo dos determinantes da evolução da relação peso para altura e peso para idade, optou-se por modelagem linear multivariada (16): foram estudados simultaneamente os efeitos dos vários fatores assumidos como potencialmente explicativos. As variáveis explicativas (independentes) ligadas à criança foram peso ao nascer em gramas; doença no pós-natal imediato (sim ou não); hospitalização pós-alta da maternidade (sim ou não); doença nos últimos 15 dias antes da observação (sim ou não); problema permanente de saúde (sim ou não); sexo; idade em meses; e condições de exposição ligadas à família: escolaridade materna em categorias (analfabeta, apenas alfabetizada, ensino fundamental completo e incompleto, ensino secundário completo e incompleto, superior completo e incompleto), duração do aleitamento materno, idade da mãe em anos, presença do pai no domicílio (sim ou não) e, ainda, posse de bens duráveis (rádio, televisão, geladeira, telefone, carro e motocicleta) como indicador de nível socioeconômico da família.

Ajustaram-se os modelos de regressão (16) tendo como variáveis de efeito (dependentes) os incrementos nos valores contínuos de escore z para os indicadores peso para idade e peso para altura.

\section{RESULTADOS}

Foram alocadas para o estudo 532 crianças (196 no GI e 336 no GN). Houve 16 e 19\% de perdas de seguimento no GI e GN, respectivamente. A comparação entre as crianças que permaneceram no estudo e as que não o fizeram mostrou que os grupos eram comparáveis quanto às variáveis relativas à criança e sua família, exceto em relação à perda de crianças com idade igual ou menor do que 36 meses, a qual foi maior entre as que não permaneceram no estudo.

A tabela 1 mostra a comparação entre o GI e o GN ao início do estudo, conforme publicação anterior (12). Como já havia sido observado, os indicadores antropométricos apresentaram comportamento distinto ao longo do seguimento, com melhor desempenho do GI. Esse favorecimento da assistência manteve-se significativo mesmo depois dos procedimentos de estratificação realizados no estudo anterior (12) para controle do possível

TABELA 1. Características das crianças de 3 meses a 6 anos na primeira avaliação segundo grupo de alocação, Sorocaba (SP), Brasil, 1995

\begin{tabular}{|c|c|c|c|c|c|}
\hline \multirow[b]{3}{*}{ Característica } & \multicolumn{4}{|c|}{ Grupo } & \multirow[b]{3}{*}{$P$} \\
\hline & \multicolumn{2}{|c|}{ Intervenção (creche) } & \multicolumn{2}{|c|}{ Não intervenção } & \\
\hline & No. & $\%$ & No. & $\%$ & \\
\hline Idade $\leq 36$ meses & $51 / 164$ & 31 & $173 / 280$ & 62 & $<0,0001$ \\
\hline Sexo masculino & $83 / 164$ & 51 & $149 / 280$ & 53 & $>0,05$ \\
\hline Prematuridade & $13 / 145$ & 9 & $15 / 241$ & 6 & $>0,05$ \\
\hline \multicolumn{6}{|l|}{ Pelo menos uma hospitalização } \\
\hline desde o nascimento & $56 / 145$ & 39 & $56 / 241$ & 23 & $<0,0001$ \\
\hline Morbidade referida ${ }^{\mathrm{a}}$ & $38 / 145$ & 26 & $166 / 280$ & 59 & $<0,0001$ \\
\hline Peso ao nascer $\leq 2500 \mathrm{~g}$ & $12 / 108$ & 11 & $29 / 263$ & 11 & $>0,05$ \\
\hline Doença no berçário & $15 / 145$ & 10 & $26 / 241$ & 11 & $>0,05$ \\
\hline Peso por altura: escore $z \leq-1$ & $56 / 164$ & 34 & $33 / 167$ & 20 & $<0,0000$ \\
\hline \multicolumn{6}{|l|}{ Escolaridade materna (inferior a } \\
\hline ensino fundamental) & $120 / 145$ & 83 & $203 / 241$ & 84 & $<0,005$ \\
\hline Mãe trabalhando fora de casa & $127 / 145$ & 88 & $46 / 241$ & 19 & $<0,0001$ \\
\hline \multicolumn{6}{|l|}{ Renda familiar per capita } \\
\hline$\leq 100$ dólares & $91 / 145$ & 63 & $162 / 241$ & 68 & $>0,05$ \\
\hline Presença do pai morando em casa & $113 / 145$ & 78 & $204 / 241$ & 85 & $>0,05$ \\
\hline
\end{tabular}

a Doença nos últimos 15 dias antes da primeira entrevista. 
TABELA 2. Variações médias dos escores z segundo grupo, seqüência de avaliação e indicadores estudados, Sorocaba (SP), Brasil, 1995

\begin{tabular}{lcrc}
\hline & & \multicolumn{2}{c}{ Indicador $^{\mathrm{a}}$} \\
\cline { 3 - 4 } Avaliação & Grupo & Peso por idade & Peso por altura \\
\hline $1^{\text {a }}$ para $2^{\mathrm{a}}$ & Intervenção (creche) & $0,073 \mathrm{a}$ & $0,580 \mathrm{~b}$ \\
& Não intervenção & $0,004 \mathrm{a}$ & $0,030 \mathrm{a}$ \\
$2^{\mathrm{a}}$ para 3 & $0,250 \mathrm{a}$ & $0,170 \mathrm{~b}$ \\
& Intervenção & $-0,060 \mathrm{~b}$ & $0,060 \mathrm{a}$ \\
$3^{\text {a }}$ para 4 & Não intervenção & $-0,010 \mathrm{a}$ & $0,140 \mathrm{a}$ \\
& Intervenção & $0,190 \mathrm{~b}$ & $0,050 \mathrm{~b}$ \\
$1^{\text {a }}$ para 4 & Não intervenção & $0,290 \mathrm{a}$ & $0,590 \mathrm{~b}$ \\
& Intervenção & $0,150 \mathrm{a}$ & $0,910 \mathrm{a}$ \\
\hline
\end{tabular}

a Letras diferentes indicam diferenças estatisticamente significativas $(P<0,05)$, sendo os maiores incrementos representados por "b".

b Foram realizadas quatro avaliações, com intervalo de 3 meses entre si.

efeito das diferenças entre os grupos, considerando o estado nutricional ao início do estudo (análise feita a partir de estratos de escores $z$ de peso para altura e peso para idade) e freqüência de crianças mais jovens no GN. Entre as crianças assistidas, $31 \%$ experimentaram incremento do escore $\mathrm{z}$ para adequação do peso para altura, enquanto a proporção entre os não assistidos foi de apenas $9 \%$, considerando o intervalo entre a primeira e quarta observações. O incremento do escore $\mathrm{z}$ para adequação do peso para altura foi de $19 \%$ (GI) e $13 \%$ (GN) entre crianças menores de 2 anos e de $33 \%$ (GI) e $7 \%$
(GN) entre aquelas com idades superiores (12).

A tabela 2 mostra resultados equivalentes aos descritos anteriormente quando são analisados os incrementos médios dos valores de escore $\mathrm{z}$, fato também mais evidente ao se considerar o índice peso por altura. Os dados na tabela permitem observar que a evolução das médias do escore $\mathrm{z}$ dos indicadores antropométricos foi sempre positiva para as crianças do GI entre uma observação e outra, exceto da terceira para a quarta observação. Os dados mostram também que essa evolução favorável é mais evidente quando se usa a relação peso para altura. Entre a primeira e a segunda observações, houve acréscimo de 0,58 ao valor do escore $\mathrm{z}$ de peso para altura no GI, contra apenas 0,03 no GN. A partir de então, o GN passou a ter redução na média do escore $\mathrm{z}$ para peso/altura, sendo as diferenças entre os grupos estatisticamente significativas. As diferenças observadas tanto intra quanto inter grupos foram menos expressivas quando se considera o indicador peso para idade. Buscaramse, sem sucesso, explicações plausíveis para o comportamento dos indicadores antropométricos entre a terceira e quarta observações, tais como erro sistemático de medidas por defeito de equipamento e falha de entrevistador na aferição antropométrica ou no registro e digitação das informações.

Apesar das condições sociais precárias e dos antecedentes de saúde desfavoráveis, a análise dos determinantes do estado nutricional das crianças ao início do estudo, portanto na ausência da intervenção para ambos os grupos, mostrou que apenas peso ao nascer apresentou significância estatística na associação entre condições antecedentes das crianças e seu estado nutricional (tabela 3).

Por outro lado, tal influência do peso ao nascer sobre o estado nutricio-

TABELA 3. Estimativa do efeito ${ }^{a}$ de cada possível causa de variação dos indicadores peso por idade e peso por altura na primeira avaliação, Sorocaba (SP), Brasil, 1995

\begin{tabular}{|c|c|c|c|c|c|c|c|c|}
\hline \multirow[b]{3}{*}{ Causa } & \multicolumn{4}{|c|}{ Grupo de intervenção (creche) } & \multicolumn{4}{|c|}{ Grupo de não intervenção } \\
\hline & \multicolumn{2}{|c|}{ Peso por idade } & \multicolumn{2}{|c|}{ Peso por altura } & \multicolumn{2}{|c|}{ Peso por idade } & \multicolumn{2}{|c|}{ Peso por altura } \\
\hline & $F$ & $P$ & $F$ & $P$ & $F$ & $P$ & $F$ & $P$ \\
\hline Sexo & 0,24 & 0,63 & 2,33 & 0,13 & 3,65 & 0,13 & 0,49 & 0,48 \\
\hline Idade & - & - & 0,038 & 0,84 & - & - & 0,46 & 0,53 \\
\hline Peso ao nascer & 10,24 & 0,00 & 5,10 & 0,03 & 15,23 & 0,00 & 6,07 & 0,02 \\
\hline Doença ao nascer & 0,18 & 0,68 & 0,04 & 0,84 & 0,47 & 0,49 & 0,88 & 0,35 \\
\hline Hospitalização após alta & 0,32 & 0,57 & 0,03 & 0,86 & 0,64 & 0,42 & 0,12 & 0,73 \\
\hline Doença nos últimos 15 dias & 0,19 & 0,67 & 0,25 & 0,62 & 4,83 & 0,12 & 3,01 & 0,10 \\
\hline Problema permanente de saúde & 0,43 & 0,51 & 1,73 & 0,19 & 2,29 & 0,13 & 0,56 & 0,46 \\
\hline Aleitamento materno ${ }^{b}$ & 3,56 & 0,14 & 3,56 & 0,10 & 0,02 & 0,88 & 0,24 & 0,62 \\
\hline Escolaridade da mãe & 0,08 & 0,78 & 0,57 & 0,45 & 0,00 & 0,99 & 0,12 & 0,73 \\
\hline Soma dos bens ${ }^{c}$ & 2,28 & 0,13 & 3,67 & 0,11 & 1,41 & 0,24 & 0,00 & 0,95 \\
\hline Idade da mãe & 0,98 & 0,33 & 0,13 & 0,72 & 0,27 & 0,61 & 0,39 & 0,53 \\
\hline Presença do pai morando em casa & 0,34 & 0,56 & 0,52 & 0,47 & 2,48 & 0,12 & 3,16 & 0,08 \\
\hline
\end{tabular}


TABELA 4. Estimativa do efeito ${ }^{a}$ das possíveis causas de variação dos indicadores peso por idade e peso por altura comparando quatro observações sucessivas, Sorocaba (SP), Brasil, 1995

\begin{tabular}{|c|c|c|c|c|c|c|c|c|c|c|c|c|}
\hline \multirow{3}{*}{ Causa } & \multicolumn{12}{|c|}{ Comparações das observações } \\
\hline & \multicolumn{4}{|c|}{ Primeira para segunda } & \multicolumn{4}{|c|}{ Segunda para terceira } & \multicolumn{4}{|c|}{ Terceira para quarta } \\
\hline & \multicolumn{2}{|c|}{ Peso por idade } & \multicolumn{2}{|c|}{ Peso por altura } & \multicolumn{2}{|c|}{ Peso por idade } & \multicolumn{2}{|c|}{ Peso por altura } & \multicolumn{2}{|c|}{ Peso por idade } & \multicolumn{2}{|c|}{ Peso por altura } \\
\hline Freqüência à creche & 3,08 & 0,08 & 26,26 & 0,00 & 31,57 & 0,00 & 14,90 & 0,00 & 5,23 & 0,02 & 4,31 & 0,05 \\
\hline Sexo & 2,90 & 0,09 & 2,69 & 0,10 & 0,53 & 0,47 & 4,46 & 0,14 & 3,47 & 0,06 & 0,63 & 0,43 \\
\hline 15 dias & 4,27 & 0,04 & 0,18 & 0,67 & 0,10 & 0,76 & 0,07 & 0,78 & 0,25 & 0,62 & 1,66 & 0,20 \\
\hline
\end{tabular}

a Significância do impacto da variável (estatística F).

nal deixa de ser significativa ao longo do seguimento quando se consideram os dois grupos no mesmo modelo, e acrescentando, neste modelo, a condição de a criança ter sido ou não sujeita à intervenção. A partir, portanto, da inclusão desta variável, a mesma passa a explicar a variação nos indicadores antropométricos, ajustando-se para as condições que expressavam, no momento da seleção, diferenças entre os grupos. A tabela 4 mostra que a evolução dos valores de escore $z$ para peso por idade e peso por altura entre observações sucessivas é de fato determinada pela intervenção (creche). O impacto da assistência sobre o estado nutricional é sempre maior (estimadores de efeito mais altos) e mais imediato, já nos primeiros 3 meses de intervenção, quando se considera a adequação do peso para altura. $\mathrm{O}$ mesmo comportamento dos indicadores acontece na evolução entre a primeira e a última observação (tabela 5).

\section{DISCUSSÃO}

Controlados os possíveis fatores de confusão ou modificadores de resultados anteriormente descritos, o presente trabalho confirma informações provenientes de comunicação anterior (17) quanto à influência decisiva do peso ao nascer como única variável significativa antes da entrada da criança na creche. Da mesma forma, estudo do crescimento de 200 crianças

TABELA 5. Estimativa do efeito das causas de variação do peso por idade e peso por altura na comparação entre a primeira e a quarta observações, Sorocaba (SP), Brasil, 1995

\begin{tabular}{lcccc}
\hline & \multicolumn{2}{c}{ Peso por idade } & & \multicolumn{2}{c}{ Peso por altura } \\
\cline { 2 - 5 } \multicolumn{1}{c}{ Causa } & $F$ & $P$ & $F$ & $P$ \\
\hline Sexo & 0,10 & 0,88 & 2,87 & 0,09 \\
Idade & - & - & 0,25 & 0,66 \\
Freqüência à creche & 0,23 & 0,63 & 33,25 & 0,00 \\
Peso ao nascer & 0,98 & 0,34 & 0,33 & 0,62 \\
Doença de berçário & 0,07 & 0,84 & 0,34 & 0,58 \\
Hospitalização após alta & 0,78 & 0,36 & 0,05 & 0,85 \\
Doença nos últimos 15 dias & 0,00 & 0,92 & 0,05 & 0,82 \\
Problema permanente de saúde & 3,78 & 0,06 & 1,04 & 0,31 \\
Aleitamento materno & 1,71 & 0,17 & 0,63 & 0,42 \\
Escolaridade da mãe & 0,79 & 0,43 & 0,94 & 0,28 \\
Renda per capita & 3,33 & 0,14 & 0,99 & 0,28 \\
Idade da mãe & 0,10 & 0,75 & 0,50 & 0,42 \\
Moradia da mãe com o pai & 0,01 & 0,96 & 0,63 & 0,46 \\
\hline
\end{tabular}

${ }^{a}$ Significância do impacto da variável (estatística F)

da zona urbana de Botucatu, Estado de São Paulo, do nascimento até 2 anos de idade, estratificadas em quatro coortes subseqüentes (18), evidenciou que um terço delas apresentaram risco de desnutrição associado a déficit ponderal ao nascer, além de outras intercorrências pós-natais. Além disso, Horta et al. (19) e Menezes et al. (20) constataram, em duas coortes de base populacional no sul do país, que o baixo peso ao nascer implica em risco 12 vezes maior de mortalidade no $1^{\mathrm{o}}$ ano de vida em comparação ao risco de mortalidade para lactentes com peso satisfatório ao nascimento. No presente estudo, essa condição dá lugar à freqüência à creche como variável expli- cativa do melhor crescimento verificado entre as crianças assistidas.

A influência favorável da assistência em creche sobre perfil de crescimento no grupo estudado permanece após controle de variáveis de confusão e sugere que os benefícios da creche são maiores do que os riscos a ela associados. Recentemente, outros autores (21) observaram, em população semelhante, que tanto o estado nutricional quanto os níveis de hemoglobina são positivamente influenciados pela freqüência à creche. $\mathrm{O}$ oferecimento, na instituição aqui estudada, de dieta balanceada, constituída por alimentos na sua maioria in natura, respeitando os hábitos locais e atendendo 
a praticamente $100 \%$ dos requisitos energéticos, protéicos e de micronutrientes (22), pode explicar o impacto positivo aqui demonstrado.

Cumpre ainda assinalar que o ambiente das creches possui outras condições que favorecem o crescimento e desenvolvimento das crianças atendidas. Powell et al. (23), em estudo com população da mesma faixa etária em área rural da América Central, já tinham relevado a necessidade de ações integradas voltadas à saúde e educação. A atividade física estimulada, envolvendo jogos e desafios, como caminhadas, corridas e subidas, foi identificada por Torun e Viteri (24) como elemento favorável ao crescimento em crianças dos 2 aos 4 anos de idade, comparadas com respectivos controles, reconhecendo, no processo, provável mediação endócrina de fatores de crescimento com síntese facilitada pelo exercício: ambos os grupos apresentaram ganhos de peso equivalentes, mas alturas superiores foram alcançadas mais precocemente pelos primeiros.
No presente estudo, diferenças de distribuição de idade entre os grupos, com maior freqüência de crianças mais jovens no GN e prevalência mais alta de escores $\mathrm{z}$ menores do que 1 no GI, recomendam cautela na interpretação do impacto da intervenção aqui verificado. Essas condições poderiam influenciar os resultados, tendo em vista a tendência em direção à média que se observa quando são feitas medidas repetidas ao longo do tempo (11). Entretanto, as análises descritivas da evolução dos indicadores nutricionais, realizadas a partir de estratificação em valores de escore $z$ ao início da observação e, também, a partir de estratificação etária (12), minimizam tal possibilidade e dão suporte à conclusão de que as diferenças observadas na evolução antropométrica entre os grupos ocorreram independentemente do estado nutricional e da idade ao início do estudo.

Apesar do impacto positivo no crescimento físico verificado neste e em outros estudos, a oferta de assistência em creches a crianças menores de 6 anos continua extremamente insuficiente, levando mães e familiares a buscarem alternativas de cuidado às suas próprias custas (25). Conforme a Fundação Sistema Estadual de Análise de Dados (SEADE) (26), em São Paulo apenas $25 \%$ dos pré-escolares encontram-se atendidos, sendo de $27 \%$ a cobertura em Sorocaba, o que demonstra o distanciamento do estado brasileiro do cumprimento de suas responsabilidades em relação a necessidades básicas da população.

Em síntese, instituições apropriadas para o cuidado em idade pré-escolar são um imperativo de ordem social no Brasil, tanto pelas razões expostas quanto pelas necessidades ligadas à crescente proporção de mulheres que buscam o mercado de trabalho (27); estima-se que $24 \%$ das famílias são, atualmente, chefiadas por mulheres (28). É obrigação governamental, em todos os níveis, prover políticas públicas (29) dirigidas a estratos populacionais que enfrentam a diminuição das oportunidades de emprego e renda.

\section{REFERÊNCIAS}

1. Fundo das Nações Unidas para a Infância (UNICEF). Situação mundial da infância, 1998. Brasília, DF: UNICEF; 1999.

2. Monteiro CA. Velhos e novos males da saúde no Brasil: a evolução do país e de suas doenças. São Paulo: Hucitec; 1995.

3. Barros RP, Mendonça R, Rocha S. Brazil: welfare, inequality, poverty, social indicators and social programs in the 1980s. Em: Lustig N, ed. Coping with austerity. Washington: Bookings Institution; 1995. Pp. 57-65.

4. Pinstrup-Andersen P. Macroeconomic adjustment policies and human nutrition: available evidence and research needs. Food Nutr Bull 1987;9(1):69-86.

5. Peliano AM. Quem se beneficia dos programas governamentais de suplementação alimentar? Brasília: Instituto de Pesquisa Econômica Aplicada (IPEA), Divisão de Editoração e Divulgação; 1990.

6. Oliveira JED, Cunha SFC, Marchini JS. A desnutrição dos pobres e dos ricos: dados sobre a alimentação no Brasil. São Paulo: Sarvier; 1996.

7. Brasil, Ministério da Saúde. Portaria $n^{\circ} 710$, de 10 de junho de 1999. Brasília: Diário Oficial da União; 11/06/99, nº 110 E, Seção I, Pp. 1419; 1999.

8. Dall'aqua FM. O ajustamento econômico e as políticas sociais: o caso do programa de merenda escolar. Em: Campino ACC, Amaral
CM, eds. Questões sociais no Brasil. São Paulo: Instituto de Pesquisas Econômicas/ Universidade de São Paulo; 1994. Pp. 57-77.

9. Barros AJD, Gonçalves EY, Borba CRS, Lorenzatto CS, Motta DB, Silva VRL, et al. Perfil das creches de uma cidade de porte médio no sul do Brasil: operação, cuidado, estrutura física e segurança. Cad Saude Publica 1999;15(3): 597-604.

10. Fuchs SC, Maynart RC, Costa LF, Cardozo A, Schierhot R. Duration of day-care attendance and acute respiration infection. Cad Saude Publica 1996;12(3):291-296.

11. World Health Organization. Report of the WHO Expert Committee on Physical Status: the use and interpretation of anthropometry. Geneva: WHO; 1995. (Technical Report Series 854).

12. Segall-Corrêa AM, Gonçalves NNS, Gonçalves A, Leite GPR, Padovani CR. Evolução da relação entre peso e altura e peso e idade em crianças de 3 meses a 6 anos assistidas em creche, Sorocaba, SP, Brasil. Rev Panam Salud Pub 1999;6(1):26-33.

13. Campbell DT, Stanley JC. Delineamentos experimentais e quase experimentais de pesquisa. São Paulo: Editora Pedagógica e Universitária/EDUSP; 1979

14. Cameron N. Assessing the nutritional status of young children in household surveys: how to weigh and measure children. New York: United Nations; 1986.

15. Gonçalves NNS, Segall-Corrêa AM, RussoLeite GP, Padovani CR, Gonçalves A. Estudo quase-experimental de impacto nutricional do programa de avaliação em creches do município de Sorocaba, SP: plano metodológico, controle de vieses e confundimento. Belo Horizonte: $49^{a}$ Reunião Anual da SBPC; 1997.

16. Searle SR. Linear models. New York: John Wiley \& Sons; 1971.

17. Segall-Corrêa AM, Russo leite GP, Padovani CR, Gonçalves NNS, Gonçalves A. Evolução do estado nutricional de crianças de 3 a 72 meses de idade assistidas em creches, Sorocaba, SP: abordagem de determinantes. Aguas de Lindóia: V Congresso Brasileiro de Saúde Coletiva; 1997.

18. Carvalhaes MABL. Crescimento nos primeiros anos de vida na área urbana do Município de Botucatu, SP. Campinas: Anais I Congresso Brasileiro de Epidemiologia Abrasco; 1990.

19. Horta BL, Barros FC, Halpern R, Victora CG. Baixo peso ao nascer em duas coortes de base populacional no sul do Brasil. Cad Saude Publica 1996;12(supl 1):27-31.

20. Menezes AMB, Victora CG, Barros FC, Albernaz G, Menezes F, Jannke HA, et al. Mortalidade infantil em duas coortes de base populacional no sul do Brasil: tendências e 
diferenciais. Cad Saude Publica 1996;12(supl 1):79-86.

21. Souza PC, Taddei JAAC. Efeito da freqüência à creche nas condições de saúde e nutrição de pré-escolares residentes em favelas da periferia de São Paulo-1996. Rev Paulista Pediatria 1998;16(3):143-150.

22. Russo-Leite GP. Consumo alimentar e o impacto no estado nutricional de pré-escolares de uma creche pública de Sorocaba, SP [dissertação de mestrado]. Campinas: Faculdade de Engenharia de Alimentos/Unicamp; 1999.

23. Powell CA, Walker SP, Chang SM, GranthamMcgregor S. Nutrition and education: a randomized trial of the effects of breakfast in rural primary school children. Am J Clin Nutr 1998;68(4):873-879.
24. Torun B, Viteri FE. Influence of exercise on linear growth. Eur J Clin Nutr 1994;48(suppl 1):186-190.

25. Ometo AMH, Furtuoso COM, Silva MV. Economia brasileira na década de oitenta e seus reflexos nas condições de vida da população. Rev Saude Publica 1995;29(5):403-414.

26. Sampaio Y, Campino AC. Food and nutrition in Brazil. Food Nutr Bull 1991;13(3):190-201.

27. Fundação Instituto Brasileiro de Geografia e Estatística (IBGE). Pesquisa nacional por amostra de domicílios: síntese de indicadores 1996. Rio de Janeiro: IBGE; 1997.

28. Barros AJD. Health risks among child day care centre attenders: the role of day care centre characteristics in common childhood illness [doctoral dissertation]. London: Univer- sity of London/Faculty of Medicine/London School of Hygiene and Tropical Medicine; 1996.

29. Fundação Sistema Estadual de Análise de Dados (SEADE) [site da Internet]. Disponível em http://www.seade.gov.br/Produtos/Informações dos municípios paulistas. Acessado em 8 de março de 2002.

Manuscrito recebido em 30 de julho de 2001. Aceito em versão revisada em 10 de janeiro de 2002.

ABSTRACT Objective. To assess factors determining growth in a group of children between 3 months and 6 years old enrolled in a public municipal (i.e., government-supported, not private) day-care center, in comparison to a group of children with similar characteristics but who were not enrolled in the center.

Factors determining weight and height in children aged 3 months to 6 years enrolled in a public municipal Methods. A quasi-experimental study was designed to observe 444 children aged 3 to 72 months from a low-income neighborhood in the city of Sorocaba, in the state of São Paulo, Brazil. Two groups were studied: 164 children enrolled in a local municipal day-care center (intervention group) and 280 not receiving care at the center (nonintervention, comparison group) but instead being cared for at home. Both groups were seen four times over a period of 16 months. At each observation session, the children's weight and height were measured. Information was also collected on the mother's sociodemographic characteristics and the illnesses she had suffered as well as the child's weight and other health characteristics at birth, the child's illnesses in the 15 days before each observation, and any hospitalizations.

Results. The children in both groups were from low-income families, with $65 \%$ of the families having an average monthly income below US\$100;80\% of the mothers had received 8 years of schooling or less. Multivariate linear regression analysis showed that at the first observation (just before enrollment in the day-care center), birth weight was the only factor that explained the nutritional differences between the two groups. Subsequent analyses showed that being in day care was the factor that best explained the differences between the groups, especially in terms of the adequacy of weight for age, after controlling for birthweight, sex, age at the beginning of the study, and illnesses in the 15 days before an observation session. The nutritional impact of the intervention was significant as early as 3 months after being enrolled in day care. Conclusions. The nutritional benefits of the care provided at the center outweighed the negative effects sometimes seen in such centers, such as the greater morbidity that children in day-care centers often experience in comparison to children receiving care at home. 